\title{
Recurrent stroke associated with cannabis use
}

\section{Mateo, A Pinedo, M Gomez-Beldarrain, J M Basterretxea, J C Garcia-Monco}

Drug misuse represents a risk factor for cerebrovascular disease, especially among young people. Despite the fact that cannabis is the most widely used illicit drug, there are only a few reports associating its use with cerebrovascular disease. We describe a patient who suffered three ischaemic strokes immediately after cannabis consumption. Other stroke aetiologies were ruled out, and neuroimaging revealed infarcts in different arterial areas as well as evidence of non-atherosclerotic arterial disease, which suggests an underlying vasculopathy of uncertain (toxic or inflammatory) origin. Cannabis use may be associated with ischaemic stroke in young patients, but its mechanism is unclear.

$\mathrm{D}$ rug misuse is a risk factor for stroke, especially among young people, and the substances most frequently implicated are cocaine, heroin, amphetamines, and other sympathomimetic agents. ${ }^{1}$ Despite the fact that cannabis is the most widely consumed illicit drug worldwide, it has only exceptionally been associated with cerebrovascular disease. We describe a patient with three recurrent strokes temporally related to hashish smoking.

\section{CASE REPORT}

A 36 year old primary school teacher, right handed, male, with no known vascular risk factors or migraine history, who had consumed hashish sporadically in the past, developed, after an unusually heavy hashish consumption and 3-4 alcoholic drinks at a party, an acute episode of isolated aphasia followed a few hours later by a convulsive seizure. He did not consume any other illicit drugs or medication, and only sporadically consumed alcohol (2-3 drinks on occasional weekends).

The patient's blood pressure was 120/80 mmHg on admission. Cranial MRI revealed two acute ischaemic infarcts, one over the left temporal lobe, consistent with his neurological deficit, and another area of silent ischaemia in the right parietal lobe. Magnetic resonance angiography (MRA) of the intracranial vasculature revealed a diminished calibre of the distal temporal branches of the left middle cerebral artery (MCA) without involvement of its proximal segment (fig la). MRA of the extracranial neck vasculature was normal, and no evidence of diffuse atherosclerotic disease was present. Urine toxicological screening on admission was positive for cannabis and negative for amphetamines, cocaine, methadone, opiates, benzodiazepines, and barbiturates. Serum glucose, creatinine, sodium, potassium, liver enzymes, blood count and differential, coagulation times, anticardiolipin antibodies, lupus anticoagulant, $\mathrm{C}$ and $\mathrm{S}$ proteins, antithrombin III levels, erythrocyte sedimentation rate, $\mathrm{C}$ reactive protein, venereal disease research laboratory slide test, HIV serology, and biochemical and microbiological cerebrospinal fluid examination gave normal or negative results. Transoesophageal echocardiogram, including agitated saline administration and provocative manoeuvres, was normal. Seizures did not recur and repeated electroencephalograms were normal; antiepileptic therapy was not initiated.

The patient was treated with ticlopidine. Despite therapy, and advice against drug consumption, he suffered another episode of aphasia and right hemiparesis a year later, immediately after hashish smoking at another party. His blood pressure at the emergency room was 140/80. Between the two episodes, he denied having consumed cannabis and he had been normotensive. MRI disclosed an acute left frontal cortical infarction, responsible for his symptoms, as well as another area of silent acute ischaemia over the contralateral frontal lobe. Repeated head and neck MRA studies did not reveal any additional abnormalities compared with the first study; the known diminished calibre of the distal temporal branches of the left MCA was still present. Again, a repeated diagnostic investigation gave only positive results for the presence of cannabis in urine.

The patient abstained from drug use for another $1 \frac{1}{2}$ years, then, after another heavy intake of hashish and 3-4 drinks of alcohol, he suffered from auditory agnosia. His blood pressure was $110 / 75 \mathrm{mmHg}$ on admission. MRI disclosed the presence of an acute infarct involving the right posterior temporal and lower parietal lobes and evidenced previous infarcts (fig lb-d). It disclosed no new changes compared with the first study. The patient recovered and has since ( 2 years after the last stroke) remained stable, on therapy with clopidogrel and denies further drug consumption.

\section{DISCUSSION}

Cannabis ingestion can result in a variety of side effects. The most frequent are psychiatric reactions, including behavioural abnormalities and an increased risk of schizophrenia. ${ }^{2}{ }^{3}$ Less known but no less important are its cardiovascular effects, which include tachycardia, ${ }^{4}$ hypertension, especially in decubitus, ${ }^{5}$ and postural hypotension. ${ }^{6}$ Carboxy-haemoglobin levels are also increased, thus decreasing the oxygen transportation capacity. ${ }^{7}$ Furthermore, cannabis increases the risk of myocardial infarction 4.8-fold during the hour following its intake ${ }^{8}$ and has been associated with paroxysmal atrial fibrillation, ${ }^{9}$ and there are reports of sudden death of cardiovascular origin following its consumption. ${ }^{10}$ Despite the fact that cannabis is the most consumed illicit drug, only 15 patients have so far been described with strokes associated to cannabis consumption. ${ }^{11-21}$ In our patient the most relevant data were the presence of three repeated strokes in close temporal relationship with cannabis and alcohol intake, involving different arterial territories in the absence of an embolic source or atherosclerotic disease, and the favourable outcome after cessation of drug consumption.

Establishing a causal relationship between drug consumption and stroke is not easy, and this relationship is usually based on a temporal link between drug use and the occurrence of stroke in young patients without any other

Abbreviations: MCA, middle cerebral artery; MRA, magnetic resonance angiography 

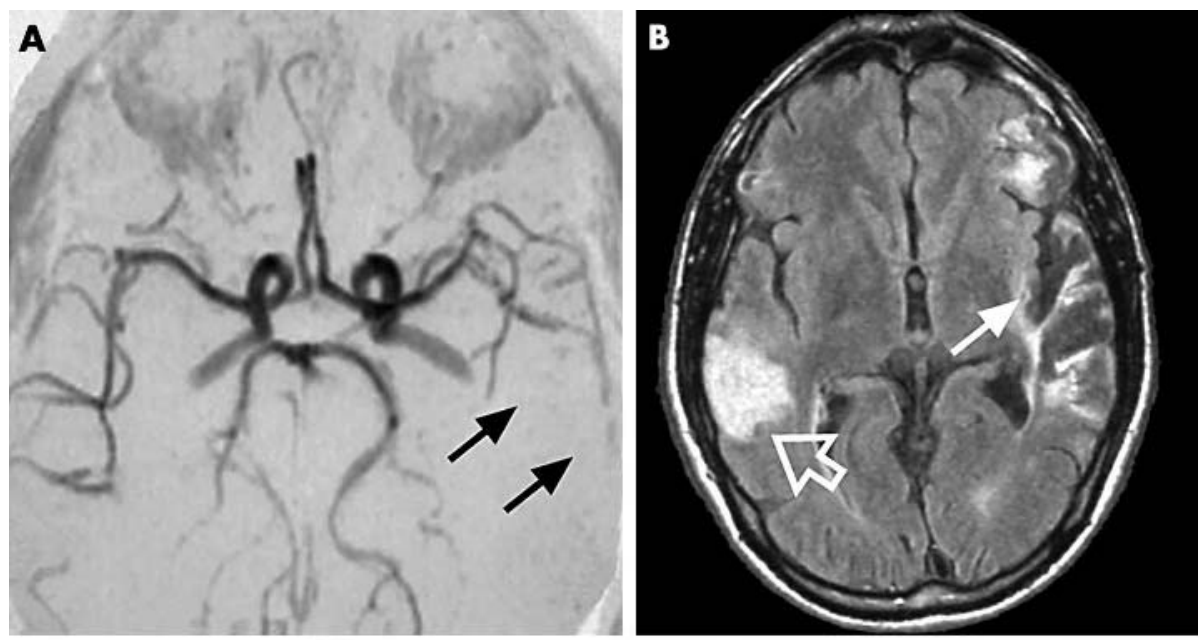

Figure 1 (a) MR angiography of this patient after his first stroke. Arterial occlusion of the distal portions of the middle cerebral artery can be seen (black arrows). (b-d) Axial FLAIR MRI sequences obtained after the last stroke. An acute ischaemic infarct is observed over the right temporal and parietal lobes (b, open arrow). Old infarcts in an atrophic stage are also evident (white arrows).
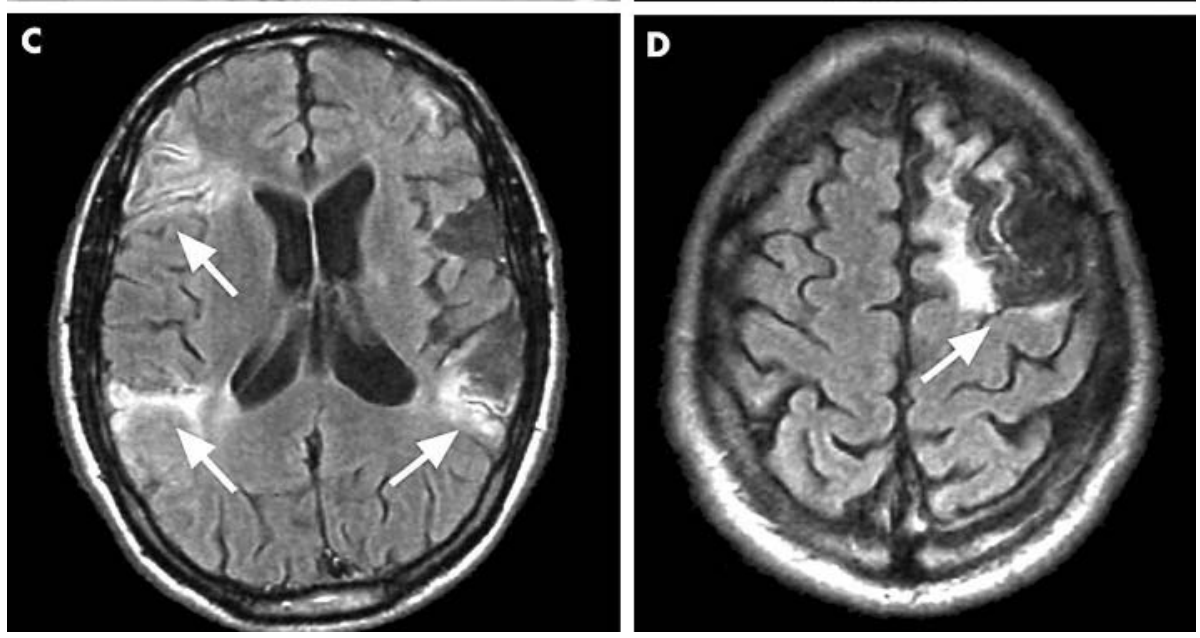

vascular risk factors. Because cannabis use is so widespread, this could represent a casual relationship; in fact, due to their high liposolubility and persistence in fatty tissues, cannabis metabolites persist in urine for weeks and therefore, their presence in a patient with a stroke could merely represent prior use. This patient's case, however, is exceptional in that there was a close and clearcut temporal relationship between drug intake and stroke, which occurred on three separate occasions. The repetition of events after exposure to cannabis, previously undescribed, suggests a role for cannabis in this patient's strokes. Alcohol ingestion could also have contributed. The relationship between alcohol and ischaemic stroke is complex and whether binge drinking is associated with stroke is controversial..$^{22}$ Nevertheless, the role of alcohol in this patient's strokes was probably secondary, as he did not consume large amounts of the drug nor much higher levels than he was used to consuming occasionally on weekends. However, hashish is an illegal drug in Spain, not subject to any regulation or control, and therefore other toxic substances could have been added during its manufacture.

The mechanisms most frequently postulated in cannabis related stroke are vasospasm ${ }^{12-14}$ and postural hypotension with abnormal regulation of the brain blood flow. ${ }^{11}{ }^{16}{ }^{23}$ In our patient, there was no evidence of postural hypotension, and blood pressure measurements in the emergency room were repeatedly normal. MRI angiography revealed a persistent mild decrease of the distal branches of the left MCA, which, together with the involvement of multiple arterial territories, simultaneously suggests the existence of an underlying vasculopathy of some sort. Some of the infarcts were not accompanied by arterial irregularities, but it is possible that these abnormalities may be below the sensitivity threshold of MRI angiography and therefore go undetected. Conventional angiography might have revealed a more diffuse damage or signs consistent with arteritis, but the patient refused it. On the other hand, the narrowing of the branches of the left MCA persisted over the years, which argues against vasospasm in this patient.

Owing to the presence of multiple ischaemic infarcts, an embolic source was insistently pursued, and was ruled out on the three admissions. However, paroxysmal atrial fibrillation can occur after cannabis consumption, and the simultaneous intake of alcohol may predispose to weekend arrhythmias and stroke. Furthermore, recent studies have shown a high incidence of paroxysmal atrial fibrillation in patients with stroke of idiopathic aetiology, mainly in patients with atrial septal abnormalities. ${ }^{24}{ }^{25}$ Repeated electrocardiograms were normal upon arrival at the emergency room on the three different occasions; 24 hour Holter and invasive heart electrophysiological studies to detect atrial vulnerability were not performed. Although the possibility could not be ruled out completely that this patient's strokes were of cardioembolic origin due to a paroxysmal atrial fibrillation triggered by alcohol and cannabis consumption, and that MRA changes reflected a partial emboli recanalisation, the absence of cardiac symptoms, the normality of the echocardiographic studies, and the absence of systemic embolic events make this possibility unlikely. As mentioned, we believe that the most likely mechanism in this case was a vasculopathy, either toxic or immune inflammatory, elicited by cannabis. 
Although there are no descriptions of cerebral vasculitis in cannabis users, the presence of arteritis involving peripheral vessels has been described. ${ }^{26}$ Cannabis arteritis resembles Buerger's disease, but in the first case patients are regular cannabis users and only moderate tobacco smokers. ${ }^{26}$ Cannabis has a peripheral vasoconstrictor effect and its prolonged use could be an additive risk factor for juvenile and young adult arteritis. ${ }^{26}$

Cannabis is not as safe a drug as many believe; it can be associated with ischaemic stroke in young people by unclear mechanisms, and toxicological screening of patients with stroke should be performed and should include the determination of cannabinoids. Future studies will be needed to clarify the role of cannabis as a stroke risk factor, as it could be underestimated. If this was the case, cerebrovascular risk may increase in the future, due to an increased consumption based on the potential therapeutic benefits of cannabis ${ }^{27}$ and its possible legalisation in some countries.

\section{Authors' affiliations}

I Mateo, A Pinedo, M Gomez-Beldarrain, J M Basterretxea, J C GarciaMonco, Service of Neurology, Hospital de Galdacano, 48960

Galdacano, Vizcaya, Spain

Competing interests: none declared

Correspondence to: Dr J C Garcia-Monco, Service of Neurology, Hospital de Galdacano, 48960 Galdacano, Vizcaya, Spain; hospit05@ sarenet.es

Received 30 March 2004

In revised form 16 June 2004

Accepted 21 June 2004

\section{REFERENCES}

1 Brust JC. Clinical, radiological, and pathological aspects of cerebrovascular disease associated with drug abuse. Stroke 1993;24(Suppl):1129-133, discussion 1134-125.

2 Andreasson S, Allebeck P, Engstrom A, et al. Cannabis and schizophrenia. A longitudinal study of Swedish conscripts. Lancet 1987;2:1483-6.

3 Solowij N, Stephens R, Roffman RA, et al. Does marijuana use cause longterm cognitive deficits? JAMA 2002;287:2653-4, author reply, 2654.
4 Renault PF, Schuster $\mathrm{CR}$, Heinrich $\mathrm{R}$, et al Marihuana: standardized smoke administration and dose effect curves on heart rate in humans. Science $1971 ; 174: 589-91$

5 Johnson S, Domino EF. Some cardiovascular effects of marihuana smoking in normal volunteers. Clin Pharmacol Ther 1971;12:762-8.

6 Hollister LE. Health aspects of cannabis. Pharmacol Rev 1986;38:1-20.

7 Aronow WS, Cassidy J. Effect of marihuana and placebo-marihuana smoking on angina pectoris. N Engl J Med J 1974;291:65-7.

8 Mittleman MA, Lewis RA, Maclure $M$, et al. Triggering myocardial infarction by marijuana. Circulation 2001;103:2805-9.

9 Kosior DA, Filipiak KJ, Stolarz P, et al. Paroxysmal atrial fibrillation in a young female patient following marijuana intoxication-a case report of possible association. Med Sci Monit 2000;6:386-9.

10 Bachs L, Morland H. Acute cardiovascular fatalities following cannabis use. Forensic Sci Int 2001;124:200-3.

11 Cooles P, Michaud R. Stroke after heavy cannabis smoking. Postgrad Med J 1987;63:511.

12 Zachariah SB. Stroke after heavy marijuana smoking. Stroke 1991;22:406-9.

13 Barnes D, Palace J, O'Brien MD. Stroke following marijuana smoking. Stroke 1992;23:1381.

14 Lawson TM, Rees A. Stroke and transient ischaemic attacks in association with substance abuse in a young man. Postgrad Med J 1996:72:692-3.

15 McCarron MO, Thomas AM. Cannabis and alcohol in stroke. Postgrad Med J 1997;73:448.

16 White D, Martin D, Geller T, et al. Stroke associated with marijuana abuse. Pediatr Neurosurg 2000;32:92-4.

17 Mesec A, Rot U, Grad A. Cerebrovascular disease associated with marijuana abuse: a case report. Cerebrovasc Dis $2001 ; 11: 284-5$.

18 Marinella MA. Stroke after marijuana smoking in a teenager with factor $V$ Leiden mutation. South Med J 2001;94:1217-18.

19 Mouzak A, Agathos P, Kerezoudi E, et al. Transient ischemic attack in heavy cannabis smokers-how 'safe' is it? Eur Neurol 2000;44:42-4.

20 Alvaro LC, Iriondo I, Villaverde FJ. Sexual headache and stroke in a heavy cannabis smoker. Headache 2002;42:224-6.

21 Russmann S, Winkler A, Lovblad KO, et al. Lethal ischemic stroke after cisplatin-based chemotherapy for testicular carcinoma and cannabis inhalation. Eur Neurol 2002;48:178-80.

22 Hillbom M, Numminen H, Juvela S. Recent heavy drinking of alcohol and embolic stroke. Stroke 1999;30:2307-12.

23 Mathew RJ, Wilson WH, Tant SR. Acute changes in cerebral blood flow associated with marijuana smoking. Acta Psychiatr Scand 1989:79:118-128.

24 Rouesnel P, Babuty D, Fauchier L, et al. Comparative study of atrial vulnerability in patients with unexplained ischemic stroke or lone atrial paroxysmal fibrillation. Ann Cardiol Angeiol (Paris) 2003:52:220-5.

25 Berthet K, Lavergne T, Cohen A, et al. Significant association of atrial vulnerability with atrial septal abnormalities in young patients with ischemic stroke of unknown cause. Stroke 2000;31:398-403.

26 Disdier P, Granel B, Serratrice J, et al. Cannabis arteritis revisited-ten new case reports. Angiology 2001;52:1-5.

27 Croxford JL. Therapeutic potential of cannabinoids in CNS disease. CNS Drugs 2003;17:179-202. 